\title{
Velocity-based boundary integral equation formulation in the time domain
}

\author{
G. D. Manolis \& C. G. Panagiotopoulos \\ Department of Civil Engineering, Aristotle University of Thessaloniki, \\ Thessaloniki, Greece
}

\begin{abstract}
In this work, we present a reciprocal theorem of linear elastodynamics derived in terms of velocities instead of displacements, which seems to be better suited in relating two different elastodynamic states of an elastic region for several reasons that will be discussed latter on. As with the conventional displacement integral equation representation, using this alternative reciprocal theorem we can produce a velocity integral equation representation and then formulate a novel numerical approximation based on the boundary element method (BEM). Furthermore, a thorough stability performance analysis of the formulations arise utilizing displacement and/or velocity reciprocal theorems is presented.
\end{abstract}

Keywords: boundary elements, convolution integrals, transient elastodynamics, reciprocal theorems, velocities.

\section{Introduction}

In linear elastodynamics and their numerical approximations based on the boundary integral equation method (BIEM), a common starting point is Graffi's reciprocal theorem relating the displacement and traction fields in a given solid body [1]. Furthermore, either a variational or a weighted residual statement that leads to the above reciprocal theorem can also serve as the starting point [2].

In general, reciprocal theorems in elasticity provide a relation between displacements, tractions and body forces for two different loading states pertaining to the same solid. Assuming that the one loading state is the one sought, and another is that corresponding to a unit impulse applied at zero time and at fixed source point $\xi$ in the infinite region and in the direction of the coordinate axes, it is possible to obtain what is called an integral representation for displacements in elastodynamics. A drawback, however, of the reciprocal 
relation is that it does not include the purely elastostatics case; also, the numerical formulations derived from its use are known to present stability problems unless special precautions are taken.

The advantage of this velocity-based reciprocal theorem, as well as of the numerical approximation that derives from it is as follows:

- It includes the case of elastostatics from which the well-known Betti's reciprocal theorem may be derived.

- Problems for which the prescribed boundary data of Dirichlet-type and given as velocities at discrete time intervals may be countered without the need of time integration to recover the corresponding displacement input data.

- More stable numerical methods than those produced by the conventional displacement formulation.

It is important here to mention that velocity integral representation formula has also been presented and utilized elsewhere $[3,4]$ but not as an outgrowth of the respective reciprocal theorem and also used for other purposes, i.e. discontinuous traction field, than for these presented here that are theoretical consistency of reciprocal statement in elastodynamics and numerical stability of BIEM formulations derived.

\section{Reciprocal theorems in terms of displacements}

The reciprocal theorem first specifies a regular region $V$ in the sense of Kellog with boundary $S$ and material properties $\rho, c_{1}, c_{2}$ as the mass density, the pressure and shear velocity of propagation respectively. Considering two distinct elastodynamic states $A=\left[u_{i}, t_{i}, b_{i}\right]$ and $B=\left[u_{i}^{\prime}, t_{i}^{\prime}, b_{i}^{\prime}\right]$ defined in that region and with initial conditions,

$$
\begin{aligned}
& u_{i}(x, 0)=u_{i}^{0}(x), v_{i}(x, 0)=v_{i}^{0}(x) \\
& u_{i}^{\prime}(x, 0)=u_{i}^{\prime 0}(x), v_{i}^{\prime}(x, 0)=v_{i}^{\prime 0}(x)
\end{aligned}
$$

where $u_{i}, t_{i}, b_{i}$ are the displacement field, the tractions and body forces respectively, while $v_{i}$ is the velocity field defined as the first time derivative of displacements. Then for time $t \geq 0$

$$
\begin{aligned}
& \int_{S} t_{i} * u_{i}^{\prime} d S+\int_{V} \rho\left(b_{i} * u_{i}^{\prime}+v_{i}^{0} u_{i}^{\prime}+u_{i}^{0} v_{i}^{\prime}\right) d V \\
& \int_{S} t_{i}^{\prime} * u_{i} d S+\int_{V} \rho\left(b_{i}^{\prime} * u_{i}+v_{i}^{\prime 0} u_{i}+u_{i}^{\prime 0} v_{i}\right) d V
\end{aligned}
$$

holds true, where operation $*$ denotes Riemann's convolution, i.e.,

$$
f * g=\int_{0}^{t} f(x, t-\tau) g(x, \tau) d \tau
$$

for $t \geq 0$ and for two arbitrary functions $f$ and $g$. As it is well known the basic approach for considering static conditions in an elastic body is to take zero values for time derivatives of the displacements. Under this consideration eqn (2) would led to the following form: 


$$
\int_{S} t_{i} * u_{i}^{\prime} d S+\int_{V} \rho b_{i} * u_{i}^{\prime} d V=\int_{S} t_{i}^{\prime} * u_{i} d S+\int_{V} \rho b_{i}^{\prime} * u_{i} d V
$$

which goes on being convoluted type relation between displacement, traction fields and body forces and it can not lead to the classical Betti's reciprocal theorem for linear elastodynamics.

\section{Reciprocal theorems in terms of velocities}

There are several ways to proceed with higher order time derivatives reciprocal theorems, e.g. variational approaches, here we adopt the simplest one which is the direct differentiation in time of eqn (2). To proceed further we utilize the Leibniz's general rule for differentiation under the integral sign, or the timedifferentiation property of convolutions, which states that:

$$
\frac{d(f * g)}{d t}=\frac{d f}{d t} * g+f^{0} g=f * \frac{d g}{d t}+f g^{0}
$$

where $f^{0}$ and $g^{0}$ stand for $f$ and $g$ at time $t=0$. We focus here at the first time derivative of eqn (2) which will provide us with the reciprocal theorem of elastodynamics in terms of velocities,

$$
\begin{gathered}
\int_{S} t_{i} * v_{i}^{\prime}+t_{i} u_{i}^{\prime 0} d S+\int_{V} \rho\left(b_{i} * v_{i}^{\prime}+b_{i} u_{i}^{\prime 0}+v_{i}^{0} v_{i}^{\prime}+u_{i}^{0} a_{i}^{\prime}\right) d V= \\
\int_{S} t_{i}^{\prime} * v_{i}+t_{i}^{\prime} u_{i}^{0} d S+\int_{V} \rho\left(b_{i}^{\prime} * v_{i}+b_{i}^{\prime} u_{i}^{0}+v_{i}^{\prime 0} v_{i}+u_{i}^{\prime 0} a_{i}\right) d V
\end{gathered}
$$

where $a_{i}$ denotes the second time derivative of $u_{i}$ component, namely the acceleration's $i^{\text {th }}$ component. Also notice here that in order to achieve eqn (6) the property of convolution given by eqn (5) has been utilized. Reciprocal statement given by eqn (6) is one of convolution type relating velocities and tractions on the boundaries of two distinct elastodynamic states as well as displacements, velocities, accelerations and body forces inside the domain $V$. For the above reciprocal relation if someone assumes static conditions, i.e. zero time derivatives for displacements, would lead directly to:

$$
\int_{S} t_{i} u_{i}^{\prime 0} d S+\int_{V} \rho b_{i} u_{i}^{\prime 0} d V=\int_{S} t_{i}^{\prime} u_{i}^{0} d S+\int_{V} \rho b_{i}^{\prime} u_{i}^{0} d V
$$

which is the well-known Betti's reciprocal theorem for linear elastostatics. It is also interesting to notice that like eqn (2), which may be seen as a "convoluted" form of the principle of virtual work produced by the displacements of one state with the tractions of the other state, eqn (6) represent a "convoluted" form of the principle of virtual power produced by velocities of one state with the tractions of the other.

\section{Axial waves in the 1D rod}

In order to investigate at least the temporal properties of algorithms derived by displacement and velocity reciprocal theorems and in an effort to avoiding the spatial discretization and integration we deal here with the simplest case 
consisting of the case of axial waves in one-dimensional finite rods (homogeneous or piecewise homogeneous).

The governing equations of the problem under consideration are the equilibrium equation plus the boundary conditions at the two end of a rod with elasticity modulus $E$, mass density $\rho$ and section $A$ :

$$
\begin{aligned}
& E A \frac{\partial^{2} u}{\partial x^{2}}-\rho A \frac{\partial^{2} u}{\partial t^{2}}=0 \\
& u(x, t)=\tilde{u}(x, t) \text { on } \Gamma_{u} \\
& f(x, t)=\tilde{f}(x, t) \text { on } \Gamma_{t}
\end{aligned}
$$

The fundamental solution as well as its derivatives (temporal or spatial) for this simple problem can be achieved with standard procedures like those described in [5] and are given below.

$$
\begin{aligned}
u^{*} & =-\frac{c}{2 E A} H\left(t-\frac{|x-\xi|}{c}\right) \\
\frac{\partial u^{*}}{\partial x} & =\frac{1}{2 E A} \frac{|x-\xi|}{x-\xi} \delta\left(-t+\frac{|x-\xi|}{c}\right) \\
\frac{\partial u^{*}}{\partial t} & =-\frac{c}{2 E A} \delta\left(t-\frac{|x-\xi|}{c}\right)
\end{aligned}
$$

In the above solutions $c=\sqrt{E / \rho}$ is the velocity of wave propagation, $\delta$ is the Dirac's delta and $H$ is the Heaviside function. Although the simplicity of eqn (8) it is very suitable in order to investigate the numerical behaviour of algorithms arise applying boundary integral methodology utilizing reciprocal theorems and also has a practical rate. A boundary integral formulation in order to numerically solve eqn (30) is also presented in [6] for the exploration of 1D wave propagation analysis in layered media.

\subsection{Time discretization and BIEM formulation}

Following standard procedures of the BIEM formulations that due to space limitations are ignored, we may derive the displacement and velocity integral representation based on reciprocal theorems in terms of displacements and velocities respectively, for a rod of length $L$ :

$$
\begin{array}{r}
u(\xi, t)=\int_{0}^{t} E A u(L, \tau) u^{\prime *}(L-\xi, t-\tau) d \tau-\int_{0}^{t} E A u^{\prime}(L, \tau) u^{*}(L-\xi, t-\tau) d \tau \\
-\int_{0}^{t} E A u(0, \tau) u^{\prime *}(-\xi, t-\tau) d \tau+\int_{0}^{t} E A u^{\prime}(0, \tau) u^{*}(-\xi, t-\tau) d \tau \\
v(\xi, t)=\int_{0}^{t} E A v(L, \tau) u^{\prime *}(L-\xi, t-\tau) d \tau-\int_{0}^{t} E A u^{\prime}(L, \tau) v^{*}(L-\xi, t-\tau) d \tau \\
-\int_{0}^{t} E A v(0, \tau) u^{\prime *}(-\xi, t-\tau) d \tau+\int_{0}^{t} E A u^{\prime}(0, \tau) v^{*}(-\xi, t-\tau) d \tau
\end{array}
$$


The ()$^{\prime}$ symbol represents a spatial derivative, and $u^{*}, u^{\prime *}, v^{*}$ are the fundamental solutions of the displacement as well as its spatial and time derivatives as given by eqs (10) and (12), respectively. In order to further proceed with the numerical implementation of integral equations (13) or (14) we divide the time interval $(0, \mathrm{t})$ into $N$ equal time steps of length $h$ and assume, for the displacement, velocity and the spatial derivative of displacements, an approximation in terms of linear basis functions. The temporal approximation of any of these fields it is given as,

$$
q(t)=\sum_{n=1}^{N}\left(H\left(t-t_{n-1}\right)-H\left(t-t_{n}\right)\right)\left(\frac{t_{n}-t}{h} q_{n-1}+\frac{t-t_{n-1}}{h} q_{n}\right)
$$

where $H$ is the Heaviside function and $t_{n}=n h$. Collocating point $\xi$ over the boundary of the domain, that is points $\xi=0$ and $\xi=L$, each one of the integral equations (13) and (14) provides us with two equations. Note also that in these integral equations we have also assumed initial conditions to be zero so that respective terms to be vanished; however it is not difficult to also include these terms. Introducing temporal approximation of the type presented in eqn (15) in integrals of eqn (13) and (14), and also accomplishing analytical temporal integrations we derive equations needed in order to have a BIEM time marching formulation of the problem. Note also here that in the case of a homogeneous 1D rod of constant cross section, internal force as well as the normal stress is given as below.

$$
\begin{aligned}
& \sigma_{x}(t)=E \varepsilon_{x}(t)=E u_{, x}(t) \\
& N(t)=A \sigma_{x}(t)=E A u_{, x}(t)
\end{aligned}
$$

From eqn (16) it is clear that for this case is near the same, from a numerical point of view, when referring to force, stress, traction or the spatial displacement derivative since all these variables are derived from the displacement's spatial derivative.

\subsection{Stability analysis}

The unstable behaviour of boundary integral equation method is well documented and has been remarked since the early appearance of time domain boundary integral equations formulations [7]. In [8] a procedure in order to investigate the stability characteristics of such equations is given, based on the $\mathrm{z}$ Transformation and some answers are provided for the "intermittent instability" phenomenon. Here we will investigate the stability of such equations utilizing a method similar to the one referred as the matrix method [9] frequently utilized in order to exploit stability properties of finite differences schemes in time. According to the matrix method, if $q_{n}$ is the vector of the unknown boundary variables at discrete time $t_{n}=n h$ then it may be written as,

$$
q_{n}=c_{n} q_{0}
$$


where $c_{n}$ is the amplification matrix of step $n$. Assuming an error in the initial data $q_{0}$ we may establish the error vector $\bar{q}_{0}$. The evolution of that error in time is given similar to eqn (17) as follows.

$$
\bar{q}_{n}=c_{n} \bar{q}_{0}
$$

In the regime of BIEM time marching formulation this $c_{n}$ amplification matrix depends on time step $n$ and it is difficult to establish an explicit form of it. Instead of exploit the evolution of the error utilizing properties of the amplification matrix, e.g. spectral radius, we proceed further in a numerical manner. That is we introduce an error in initial data and we explore this error's evolution in time computed by BIEM procedures resulted utilizing displacement and velocity reciprocal theorems. Beneficial factor of this procedure is that the only parameter manifests the stability performance for the case of $1 \mathrm{D}$ wave propagation in a rod is the non-dimensional ratio sometimes encountered as the Courant-Friedrichs-Lewy (CFL) parameter and given as

$$
\beta=\frac{c h}{L}
$$

Consequently results produced are problem independent. In Table 1 , stability regions are given explicitly, for various combinations of boundary conditions, in terms of parameter $\beta$.

Table 1: $\quad$ Stability properties for varying $\beta$ parameter.

\begin{tabular}{|c|c|c|c|}
\hline $\begin{array}{c}\text { prescribed } \\
\text { variable on } i \\
\text { (start) boundary }\end{array}$ & $\begin{array}{c}\text { prescribed } \\
\text { variable on } j \\
\text { (end) boundary }\end{array}$ & $\begin{array}{c}\text { stability on } \\
\text { region } 0 \leq \beta<1\end{array}$ & $\begin{array}{c}\text { stability on } \\
\text { region } 1<\beta \leq 2\end{array}$ \\
\hline \hline$u$ & $u$ & $\begin{array}{c}\text { unstable for any } \\
\beta \neq \frac{1}{z}\end{array}$ & $\begin{array}{c}\text { unstable for any } \\
\beta\end{array}$ \\
\hline$u$ & $u^{\prime}$ & $\begin{array}{c}\text { stable for small } \\
\text { regions of } \beta\end{array}$ & stable for any $\beta$ \\
\hline$u^{\prime}$ or $v$ & $u^{\prime}$ or $v$ & $\begin{array}{c}\text { stable for any } \\
\beta \neq \frac{1}{z}\end{array}$ & stable for any $\beta$ \\
\hline
\end{tabular}

Variable $z$ appeared in table (1) stands for the positive integer numbers. As it may observed in table (1), in formulations where only velocities and/or tractions used, that means that the velocity reciprocal theorem has been utilised, the behaviour is in general stable except for the cases where the parameter $\beta$ has been chosen such to be equal to a ratio of unity with an integer number. Regarding formulations based on the displacement reciprocal theorem it seems, as it can also be seen in table (1), that are most prone to numerical instabilities. For the case of Dirichlet problems formed in terms of displacements there is appeared a "stable" behaviour for discrete values of $\beta$, however stability can 
not be guaranteed since it is almost difficult to define these values due to computer's numerical precision. Regarding the stability regions presented in the case of mixed problem when utilizing displacement formulation these are shown to have a finite range near to $\beta=1 /(z+0.5)$ and also near but not equal to the value of $1 / z$. For increasing $z$, and furthermore for decreasing $\beta$ and time step value $h$ these finite stability region are decrease and tend to degenerate to discrete values.

\section{Numerical examples}

In this section there are presented some numerical examples in order to verify the results obtained on the stability performance of algorithms utilizing the displacement as well as velocity reciprocal theorems. In the case of a single 1D rod we present a set of examples in order to cover the Dirichlet, as also the mixed type of problems. Both examples consider a rod of elasticity modulus $E=1000 \mathrm{kN} / \mathrm{m}^{2}$, mass density $\rho=1.0 \mathrm{t} / \mathrm{m}^{3}$, a cross section of area $A=16 m^{2}$ and a length $L=4 m$.

\subsubsection{A Dirichlet problem}

As a first example a Dirichlet type of problem is solved utilizing both the displacement as well as the velocity formulations. The rod is constrained on the $i$ and $j$ boundary points to prescribed motion given as

$$
\begin{aligned}
& u_{i}(t)=\cos (24 t)-1 \\
& u_{j}(t)=1.5 u_{i}(t)
\end{aligned}
$$

with the respective velocities give as

$$
\begin{aligned}
& v_{i}(t)=-24 \sin (24 t) \\
& v_{j}(t)=1.5 v_{i}(t)
\end{aligned}
$$

As a frequency of the prescribed motion have been chosen one near the first natural frequency of the constrained rod which is $\omega_{q}=q \pi c / L$ with $q$ equal to unit. In fig (1) numerical solution of the spatial derivative of displacement is plotted utilizing velocities formulation for parameter $\beta=1 / 30$ which is the unstable solution and also for $\beta=1 / 30.1$ which is the stable one.

\subsubsection{A mixed problem for wave propagation}

A benchmark problem which is very frequently utilized in order to test the performance of boundary integral equation methods in time is presented here. It consists of a rod with its $i$ boundary point constrained to have a zero motion in time while its $j$ boundary point excited by a constant force in time. First we consider the solutions computed utilizing the velocity formulation which, as could be seen in fig (2), are stable and really accurate for values of parameter $\beta$ equal to $1 / 101.50$ as well as $1 / 101.25$ but not for that of $\beta=1 / 101$. Contrary to 
the velocity formulation, the respective one utilizing the displacement reciprocity gives stable results only for the case where $\beta$ equals to $1 /(\mathrm{z}+0.5)$, or to values very close to that according also the notes given in sec (4.2). This can be ascertained by results in time, plotted in fig (2) where the unstable performance for $\beta$ equal to $1 / 101.25$ and $1 / 101.00$ are shown together with those of $\beta$ equal to $1 / 101.5$.

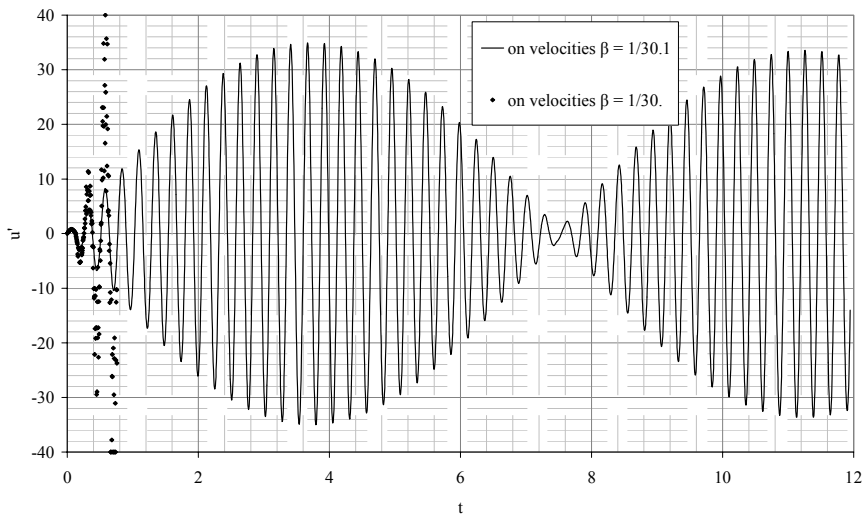

Figure 1: Displacement's spatial derivative of boundary point $i$ in time utilizing velocity formulation.

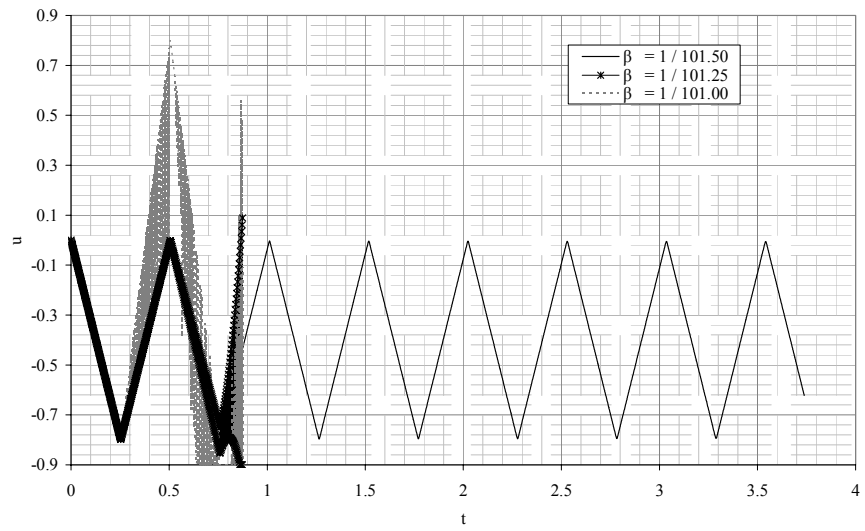

Figure 2: $\quad$ Plots of computed displacement utilizing velocity formulation.

As further verification for these almost discrete stability conditions in reference to $\beta$ parameter for the case of displacement formulation in fig (3) there is a series of solutions for various $z$ integer values. In this plot, someone may also notice the numerical damping introduced for increasing $\beta$. As also 
someone may observe in fig.(3), contrary to other 1D formulations reported elsewhere, e.g. [6], there are not presented difficulties to accurately describe discontinuous variations in time, e.g. that of tractions. Also inaccurate results as reported in [3] have as origin the discontinuity of tractions that generates an error, but their raising in time is due to unstable behaviour of the formulations.

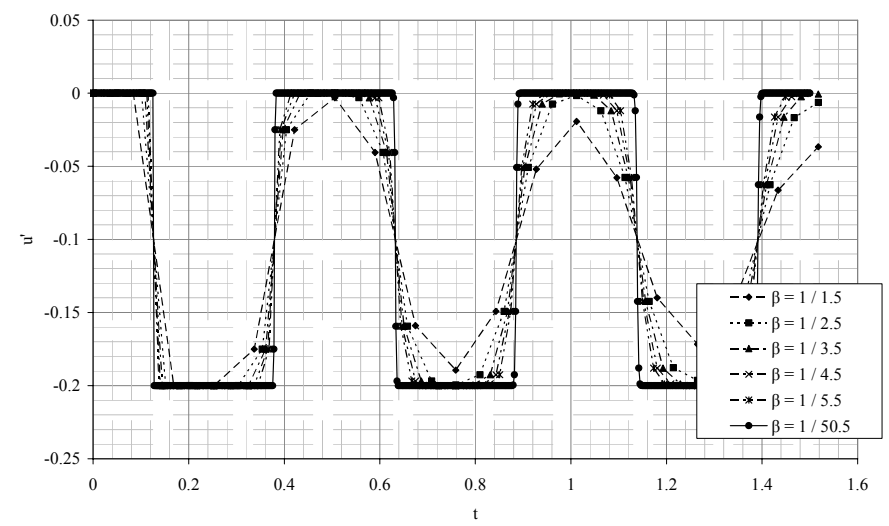

Figure 3: Displacement's spatial derivative results utilizing displacement formulation for various "stable" values of $\beta$.

\section{Conclusions}

In this work the classical Graffi's reciprocal theorem in term of displacements is re-examined as a counterpart of that of Betti's in the case of elastostatics. It is shown that, despite the doubtless validity of Graffi's theorem, nevertheless a similar reciprocal statement involving velocity field which also is valid in the case of elastodynamics, is also includes the elastostatic case as a limit case. After introducing this theorem, it is shown that it is straightforward to establish the integral representation of velocities. Furthermore utilizing this reciprocal theorem in terms of velocities, boundary integral equation methods type of formulations are established and tested on simple model problems. Also results obtained are compared to those computed utilizing conventional BIEM formulations based on the classical reciprocal theorem in terms of displacements. It is found that formulations based on velocities present a superiority regarding stability performance and considered more compatible for the case of transient elastodynamics. Also extension of the fully three-dimensional elastodynamics case with boundary elements is an ongoing research, undertaken by the authors.

\section{References}

[1] Graffi, D., Sul teorema di reciprocita nella dinamica dei corpi elastici. Memoric della Reale Accademia delle Scinze dell'Istituto di Bologna, 10, pp. 103-111, 1946. 
[2] Dominguez, J., Boundary Elements in Elastodynamics, Computational Mechanics Publications, Elsevier Applied Science, UK, 1993.

[3] Carrer, J.A.M., Mansur, W.J., Time discontinuous linear traction approximation in time-domain bem: 2-D elastodynamics. International Journal for Numerical Methods in Engineering, 49, pp. 833-848, 2000.

[4] Frangi, A., "Causal" shape functions in the time domain boundary element method, Computational Mechanics, 25, pp. 533-541, 2000.

[5] Greenberg, M.D., Applications of Green's Functions in Science and Engineering, Prentice-Hall, Inc. Englewood Cliffs, New Jersey, 1971.

[6] Moser, W., Antes, H., Beer, G., A Duhamel integral based approach to onedimensional wave propagation analysis in layered media, Computational Mechanics, 35, pp. 115-126, 2005.

[7] Mansur, W.J., A time-stepping technique to solve wave propagation problems using the boundary element method, $\mathrm{Ph} . \mathrm{D}$. thesis, University of Southampton, 1983.

[8] Pierce, A., Siebrits, E., Stability Analysis of Model Problems for Elastodynamic Boundary Element Discretizations, Numerical Methods for Partial Differential Equation, 12, pp. 585-613, 1996.

[9] Mitchell, A.R.M, Griffiths, D.F., The finite Difference Method in Partial Differential Equations, John Willey and Sons Ltd., 1988. 